\title{
Pupillometry in critically ill patients with COVID-19: a prospective study
}

\author{
Charikleia S. Vrettou ${ }^{1}$ (]) Eleni Korompoki ${ }^{2,3} \cdot$ Katerina Sarri $^{1} \cdot$ loannis Papachatzakis ${ }^{1} \cdot$ Maria Theodorakopoulou $^{4}$. \\ Evangelia Chrysanthopoulou ${ }^{4} \cdot$ Ilias A. Andrianakis $^{5} \cdot$ Christina Routsi $^{1} \cdot$ Spyros Zakynthinos $^{1} \cdot$ Anastasia Kotanidou $^{1}$
}

Received: 24 July 2020 / Accepted: 28 September 2020 / Published online: 7 October 2020

(c) Springer-Verlag GmbH Germany, part of Springer Nature 2020

Keywords Portable pupillometry $\cdot$ Autonomic nervous system · COVID-19

\section{Dear Editor,}

Since December 2019 severe acute respiratory syndrome coronavirus-2 (SARS-CoV-2) has spread over the entire world, causing the coronavirus disease 2019 (COVID-19) pandemic [1]. COVID-19 is characterized by an excessive inflammatory response [2] that could theoretically affect the pupillary light reflex (PLR) by the following mechanism: inflammatory molecules, such as cytokines, are detected by the afferent vagus nerve fibers, which terminate at the solitary nucleus; the solitary nucleus projects into the locus coeruleus, the brainstem nucleus which controls autonomic function and is the main source of norepinephrine in the brain. Locus coeruleus activation is correlated to increased pupil size, but the associated physiological mechanisms have

Electronic supplementary material The online version of this article (https://doi.org/10.1007/s10286-020-00737-7) contains supplementary material, which is available to authorized users.

Charikleia S. Vrettou

Vrettou@hotmail.com

1 First Department of Critical Care Medicine and Pulmonary Services, Evangelismos Hospital-Medical School of National and Kapodistrian University of Athens, 45-47 Ipsilantou St, 10676 Athens, Greece

2 Department of Clinical Therapeutics, Alexandra Hospital-Medical School of Athens, 80 Vass. Sofias Av., 11528 Athens, Greece

3 Division of Brain Sciences, Charing Cross Hospital-Imperial College London, Fulham Palace Road, London W6 8RF, UK

4 Second Department of Critical Care Medicine, Attikon Hospital-National and Kapodistrian University of Athens, 1 Rimini St., 12462 Athens, Greece

5 Department of Intensive Care, Ygeia Hospital, 4 Erythrou Stavrou and Kifisias Av., 15125 Athens, Greece not yet been elucidated [3]. Locus coeruleus activation also attenuates pupillary constriction via inhibitory projections to the Edinger-Westphal nucleus [4]. It is therefore plausible to expect that patients with COVID-19 have increased pupil size and decreased pupillary response to light compared to other patients with respiratory failure. Demonstrating such differences would be of clinical value because it would provide evidence for the application of pupillometry as a marker of autonomic nervous system (ANS) involvement in COVID-19 and potentially in other infectious diseases [5].

To investigate this hypothesis we measured the various parameters of the PLR of critically ill patients with COVID19 and compared these parameters with those of patients with respiratory failure of different etiology. The study took place between 20 February and 27 April 27, in the Intensive Care Unit (ICU) Department of Evangelismos General Hospital, Athens, Greece. The study was approved by the Scientific and Ethics Committee of Evangelismos Hospital, and written informed consent was obtained from all study participants or from their next of kin.

The inclusion criteria were: age $>18$ years; admission to hospital with respiratory failure requiring mechanical ventilation for $>48 \mathrm{~h}$; and informed consent from the patient or patient's next of kin. Exclusion criteria were: history, evidence or suspicion of primary neurological injury; presence of agitation or delirium; known ocular disease; disease severity that rendered treatment futile; the presence of shock requiring treatment with high-dose vasoconstrictors (dopamine $>15 \mu \mathrm{g} / \mathrm{kg} / \mathrm{min}$, or epinephrine $>0.1 \mu \mathrm{g} / \mathrm{kg} /$ $\mathrm{min}$, or norepinephrine $>0.1 \mu \mathrm{g} / \mathrm{kg} / \mathrm{min}$ ) [6]; pregnancy; and $\beta$-blocker or other anti-hypertensive drug administration at the time of evaluation. To limit the variability caused by different levels of sedation in the study population [7], we assessed the depth of sedation with the Ramsay Sedation Scale [8]. Patients who were deeply sedated (Ramsay 
score 6) and patients who were awake (Ramsay score 2) and not on any sedatives for at least $1 \mathrm{~h}$ prior to the initial assessment were included in the study. Patients with Ramsay scores ranging from 3 to 5 and patients on low-dose remifentanyl, dexmedetomidine or clonidine at the time of the initial assessment were scheduled to be re-assessed the following day. COVID-19 status was based upon positive SARS-CoV-2 real-time reverse transcriptase-PCR assay of nasopharyngeal swab specimens. For the purpose of this analysis, the patients were divided into four groups, as shown in Table 1 and in the study flowchart in the Electronic Supplementary Material (ESM).

We assessed the PLR using the NPi200 pupillometer system (NeurOptics, Laguna Hills, CA, USA) according to the manufacturer's instructions [9]. Three measurements were taken for each participant within a 30-min interval, and the average of these three measurements was used for statistical analysis. The variables recorded included the neurological pupil index (NPi), maximum pupil diameter before constriction (Size), minimum pupil diameter at peak constriction (Min), percentage of change of the pupil diameter during constriction $[\Delta$ Size $(\%)]$, pupil latency (LAT; i.e., the time-of-onset of constriction following initiation of the light stimulus), average constriction velocity (CV), maximum constriction velocity (MCV) and dilation velocity (DV). All measurements were performed under dim light conditions (5-11 lx) measured by a lux-meter (Peakmeter PM6612). Since patients with known or likely neural injury were excluded, left and right eye PLR measurements were pooled together.

We used non-parametric methods for statistical testing using IBM SPSS statistics version 25 (IBM Corp., Armonk,
NY, USA). Statistical significance was set at $p<0.05$. We included 41 patients in the analysis, 18 of whom were diagnosed with COVID-19 and 23 of whom were diagnosed with respiratory failure of a different etiology. The demographic and clinical characteristics of the study population are given in detail in the ESM. A summary of the PLR measurements and statistical comparisons between groups are given in Table 1. Data pertaining to individual patients are graphically provided in the ESM. There were no significant differences in the PLR parameters between COVID19-positive (+) and COVID-19-negative (-) patients. To assess the power of these comparisons we considered alternative hypotheses based on previously published data from PLR assessment in patients with ANS dysfunction [10]. The power for the comparisons of the MCV, assuming the alternative hypothesis that MCV is at least 34.3\% lower in patients with COVID-19, was $79.5 \%$ for sedated and $82.9 \%$ for awake patients. The effect of sedatives was far more significant than the COVID-19 status in our sample, even though not all PLR variables were equally affected by sedation. More precisely, it was pupil size, and variables that depend on pupil size [Min, $\Delta$ size (\%), CV, MCV] that were affected, as was DV. The NPi, an indicator of elevated intracranial pressure [9], did not differ significantly among the four groups. This was expected, since patients with diagnosed or likely primary neural injury were excluded.

The absence of significant differences in the PLR between the COVID-19 (+) and COVID-19 (-) patients can be attributed to several possible reasons. One possibility is that sympathetic activation is not actually different in SARS-CoV-2 infection compared to other causes of respiratory failure. Another is that even though COVID-19 affects the ANS,
Table 1 Pupillometry measurements in the different patient groups

\begin{tabular}{llllll}
\hline Variable & \multicolumn{2}{l}{ Sedated (Ramsay score 6) } & & \multicolumn{2}{l}{ Awake (Ramsay score 2) } \\
\cline { 2 - 3 } \cline { 6 - 7 } & $\begin{array}{l}\text { Group a: COVID-19 } \\
\text { negative }(n=14)\end{array}$ & $\begin{array}{l}\text { Group b: COVID-19 } \\
\text { positive }(+)(n=6)\end{array}$ & & $\begin{array}{l}\text { Group c: COVID-19 } \\
\text { negative }(n=9)\end{array}$ & $\begin{array}{l}\text { Group d: COVID- } \\
\text { 19 positive }(n=12)\end{array}$ \\
\hline NPi & $4.52(3.98-4.70)$ & $4.44(4.18-4.61)$ & & $4.53(4.45-4.55)$ & $4.64(4.39-4.71)$ \\
Size & $2.26(1.99-2.72)^{* *}$ & $2.10(2.05-2.63)^{\dagger}$ & & $3.69(3.18-4.53)^{* *}$ & $3.50(3.06-4.20)^{\dagger}$ \\
Min & $1.87(1.66-2.32)^{*}$ & $1.82(1.73-2.19)$ & & $2.60(2.27-2.73)^{*}$ & $2.33(2.05-2.80)$ \\
$\Delta$ Size $(\%)$ & $15.7(12.9-20.0)^{* *}$ & $18.2(15.6-20.1)^{\dagger \dagger}$ & & $30.7(26.5-32.8)^{* *}$ & $35.0(30.4-37.3)^{\dagger \dagger}$ \\
CV & $0.82(0.76-1.05)^{* *}$ & $0.96(0.80-1.07)^{\dagger \dagger}$ & & $1.95(1.55-2.32)^{* *}$ & $2.29(1.93-2.54)^{\dagger \dagger}$ \\
MCV & $1.36(1.11-1.52)^{* *}$ & $1.39(1.31-1.59)^{\dagger \dagger}$ & & $2.98(2.89-3.68)^{* *}$ & $3.50(3.27-4.08)^{\dagger \dagger}$ \\
DV & $0.37(0.29-0.45)^{* *}$ & $0.43(0.32-0.52)^{\dagger \dagger}$ & & $1.03(0.84-1.17)^{* *}$ & $1.03(0.91-1.17)^{\dagger \dagger}$ \\
Latency & $0.26(0.24-0.28)$ & $0.29(0.25-0.30)$ & & $0.24(0.23-0.25)$ & $0.25(0.23-0.26)$ \\
\hline
\end{tabular}

All values in table are presented as median with the interquartile range in parentheses

COVID-19 Coronavirus Disease-19, NPi neurological pupil index, Min minimum pupil size, $\Delta$ Size (\%) (Size-Min)/Size $\times 100 \%, C V$ constriction velocity, $M C V$ maximum constriction velocity, $D V$ dilation velocity

*Significant difference at $p<0.05$ (Mann-Whitney $U$ test) between groups a and c; **significant difference at $p<0.001$ (Mann-Whitney $U$ test) between groups a and c; ${ }^{\dagger}$ significant difference at $p<0.05$ (MannWhitney $U$ test) between groups $\mathrm{b}$ and $\mathrm{d}$; ${ }^{\dagger}$ significant difference at $p<0.001$ (Mann-Whitney $U$ test) between groups $\mathrm{b}$ and $\mathrm{d}$ 
this effect is attenuated in the ICU population by ICU-related interventions, such as the administration of anesthetics, analgesics and inotropic medications that may have a prolonged effect. A limitation of our study is its small sample size, due to the small number of ICU admissions with COVID-19 in Greece during the study period. Furthermore, our measurements were not blinded to the examiners, as this was not feasible given the nature of the study question.

In conclusion, PLR measurements were not significantly different between ICU patients treated for COVID-19 and patients with respiratory failure of different cause. Further prospective studies are needed to investigate whether such differences are present at earlier or later stages of the disease.

Funding No funding was received for this work.

Data availability The datasets used and/or analyzed during the current study are available from the corresponding author on reasonable request.

\section{Compliance with ethical standards}

Conflict of interest The authors declare that they have no conflict of interest.

Ethical approval The study was approved by the scientific and Ethics committee of Evangelismos Hospital.

Informed consent Written informed consent was obtained from all study participants or from their next of kin.

\section{References}

1. Munster VJ, Koopmans M, van Doremalen N, van Riel D, de Wit E (2020) A novel coronavirus emerging in China-key questions for impact assessment. N Engl J Med 382(8):692-694. https://doi. org/10.1056/NEJMp2000929

2. Huang C, Wang Y, Li X, et al (2019) Clinical features of patients infected with 2019 novel coronavirus in Wuhan, China. Lancet 395(10223):497-506. https://doi.org/10.1016/S0140 $-6736(20) 30183-5$

3. Joshi S, Li Y, Kalwani RM, Gold JI (2016) Relationships between pupil diameter and neuronal activity in the locus coeruleus, colliculi, and cingulate cortex. Neuron 89(1):221-234. https://doi. org/10.1016/j.neuron.2015.11.028

4. Samuels ER, Szabadi E (2008) Functional neuroanatomy of the noradrenergic locus coeruleus: its roles in the regulation of arousal and autonomic function part I: principles of functional organisation. Curr Neuropharmacol 6(3):235-253. https://doi. org/10.2174/157015908785777229

5. Hall CA, Chilcott RP (2018) Eyeing up the future of the pupillary light reflex in neurodiagnostics. Diagnostics 8(1):19. https://doi. org/10.3390/diagnostics8010019

6. Ferreira FL, Bota DP, Bross A, Mélot C, Vincent JL (2001) Serial evaluation of the SOFA score to predict outcome in critically ill patients. JAMA 286(14):1754-1758. https://doi.org/10.1001/ jama.286.14.1754

7. Rouche O, Wolak-Thierry A, Destoop Q, et al (2013) Evaluation of the depth of sedation in an intensive care unit based on the photo motor reflex variations measured by video pupillometry. Ann Intensive Care 3(1):5. https://doi.org/10.1186/2110-5820-3-5

8. Ramsay MA, Savege TM, Simpson BR, Goodwin R (1974) Controlled sedation with alphaxalone-alphadolone. Br Med J 2(5920):656-659. https://doi.org/10.1136/bmj.2.5920.656

9. Chen JW, Gombart ZJ, Rogers S, Gardiner SK, Cecil S, Bullock RM (2011) Pupillary reactivity as an early indicator of increased intracranial pressure: the introduction of the neurological pupil index. Surg Neurol Int 2:82. https://doi. org/10.4103/2152-7806.82248

10. Muppidi S, Adams-Huet B, Tajzoy E, et al (2013) Dynamic pupillometry as an autonomic testing tool. Clin Auton Res 23:297-330. https://doi.org/10.1007/s10286-013-0209-7 